Recepción: 09 / 11 / 2017

Aceptación: 18 / 01 / 2018

Publicación: 21 / 03 / 2018
Ciencias económicas y empresariales

Artículo de Revisión

\title{
Como se gestiona el ahorro en el Ecuador
}

\author{
How savings are managed in Ecuador
}

\section{Como as economias são gerenciadas no Equador}

\author{
Ángel C. Alvarado-Gastiaburo I \\ angel.alvaradog@ug.edu.ec \\ Nelly S. Vergara-Díaz ${ }^{\text {II }}$ \\ nelly.vergaradi@ug.edu.ec
}

Correspondencia: angel.alvaradog@ug.edu.ec

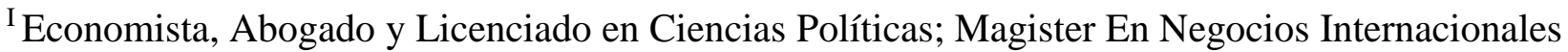
y Gestión de Comercio Exterior, Docente de la Universidad de Guayaquil, Guayaquil, Ecuador.

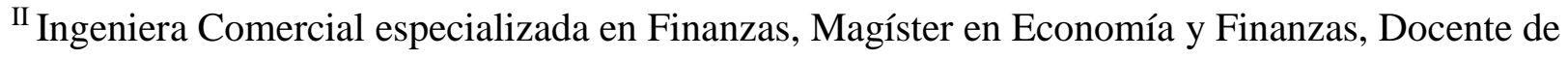
la Universidad de Guayaquil, Guayaquil, Ecuador.
} 


\section{Resumen}

Desde tiempos antiguos ya se manejaba esta actividad, aunque no con bienes económicos principalmente, el ahorro es el excedente de cualquier bien económico al final de un periodo. Existen diferentes tipos de ahorro, así como diferentes instrumentos financieros para poder ahorrar e invertir al mismo tiempo, lo pueden realizar tantas personas, familias, empresas e incluso naciones. El ahorro se puede clasificar en ahorro privado y en ahorro público.

Los cambios en la tasa de interés, por ejemplo, pueden hacer que los ahorradores se sientan motivados o desmotivados a ahorrar. Un aumento en las tasas de interés puede hacer que los ahorradores tengan más razones para disminuir su consumo y ahorrar, o bien puede tener el efecto opuesto. Las reformas económicas de los últimos años, acompañadas de una mayor estabilidad económica y política en la región, han llevado a que el universo de inversionistas y prestamistas externos con exposición a Latinoamérica sea hoy más amplio que nunca. Dentro de la economía nacional, el sector privado es la fuente principal de ahorro. Las transferencias a los hogares, incluidos los pagos de prestaciones sociales, son el cauce principal a través del cual el gasto público afecta a la acumulación de ahorro privado. En este artículo se realizó una búsqueda sistematizada de información documental y se seleccionó aquellos datos que nos permiten esbozar una síntesis del ahorro en Ecuador.

\section{Palabras clave:}

\section{Abstract}

Since ancient times this activity was already managed, although not mainly with economic goods, saving is the surplus of any economic good at the end of a period. There are different types of savings, as well as different financial instruments to be able to save and invest at the same time, so many people, families, companies and even nations can do it. Savings can be classified as private savings and public savings. Changes in the interest rate, for example, can make savers feel motivated or discouraged to save. An increase in interest rates may cause savers more reasons to decrease their consumption and save, or it may have the opposite effect. The economic reforms of recent years, accompanied by greater economic and political stability in the region, have led to a wider universe of investors and external lenders with exposure to Latin America than ever before. Within the national economy, the private sector is the main source of 
savings. Transfers to households, including payments of social benefits, are the main channel through which public spending affects the accumulation of private savings. In this article, a systematic search of documentary information was carried out and those data that allow us to sketch a synthesis of savings in Ecuador were selected.

\section{Keywords:}

\section{Resumo}

Desde a antiguidade que essa atividade já era administrada, embora não principalmente com bens econômicos, a poupança é o excedente de qualquer bem econômico no final de um período. Existem diferentes tipos de poupança, bem como diferentes instrumentos financeiros para poder poupar e investir ao mesmo tempo, para que muitas pessoas, famílias, empresas e até nações possam fazê-lo. As economias podem ser classificadas como poupança privada e poupança pública. Mudanças na taxa de juros, por exemplo, podem fazer com que os poupadores se sintam motivados ou desencorajados a economizar. Um aumento nas taxas de juros pode causar aos poupadores mais razões para diminuir seu consumo e economizar, ou pode ter o efeito oposto. As reformas econômicas dos últimos anos, acompanhadas de maior estabilidade econômica e política na região, levaram a um universo mais amplo de investidores e credores externos, com exposição à América Latina do que nunca. Dentro da economia nacional, o setor privado é a principal fonte de poupança. As transferências para as famílias, incluindo pagamentos de benefícios sociais, são o principal canal através do qual os gastos públicos afetam o acúmulo de poupança privada. Neste artigo, foi realizada uma busca sistemática de informações documentais e selecionados os dados que permitem esboçar uma síntese da economia no Equador.

\section{Palavras chave:}

\section{Introducción}

En términos de teoría económica el ahorro se refiere a la parte de la renta o ingreso no dedicada a consumo, sino a otros fines y es un concepto económico importante, existen diversas modalidades de ahorro así como diversos instrumentos financieros destinados para incrementar el ahorro que se pretende realizar, puede ser a través de algún sistema provisto por una institución 
autorizada por la ley para captar dinero del público, tal como una cuenta o tarjeta de ahorros, un depósito a plazo o una cuenta de ahorro provisional voluntario. (Superintendencia)

El ahorro es una forma del empleo o consumo de la riqueza, que consiste en no aplicar a la satisfacción de las necesidades actuales más que una parte del beneficio o retribución obtenidos en la industria, destinando el resto a aumentar los medios de que se dispone para nuevas producciones y a la atención de las necesidades futuras. (BLANCO, 1998)

Se entiende como ahorro a la parte del ingreso que no se destina al gasto y que se reserva para necesidades futuras, se puede utilizar para algún gasto importante que se tenga, algún imprevisto o emergencia económica.

\section{Características Importantes del Ahorro}

La clave del ahorro es la capacidad de juntar dinero de manera regular durante un período de tiempo, lo interesante de ahorrar en una institución financiera, es que ésta paga a las personas que le entregan su dinero. A esta ganancia se le llama rentabilidad y se expresa a través de los intereses, los cuales varían dependiendo de sus características, tales como el plazo, el tipo de ahorro y el tipo de moneda, entre otros. A estos se agregan los reajustes que permiten mantener el valor adquisitivo del dinero que se haya ahorrado, debido a que es el sistema ajusta el monto del ahorro, para mantenerlo con su mismo valor respecto de la inflación, las instituciones financieras ofrecen diferentes alternativas de ahorro, con el fin de acomodarse a la capacidad de ahorro de sus clientes. (Spencer, 1998)

El ahorro es igualmente importante para el futuro económico de cualquier nación. La producción de una empresa, por ejemplo, involucra algunos recursos que son limitados, como la tierra. Si ésta quiere mejorar su producción y tiene problemas con recursos limitados, debe buscar cómo mejorar su producción basándose en inversiones (por ejemplo, en tecnología o en máquinas). Si la empresa ahorra durante un periodo determinado, tendrá la posibilidad de acceder más fácilmente, a través de créditos, a esas maquinarias o a esa tecnología o a otros recursos económicos. Igualmente, si los bancos tienen más ahorros en las cuentas, tendrán más dinero para prestar y no será necesario que las personas, las empresas o el Estado pidan recursos en el exterior. Esto, en general, facilita e incentiva la actividad económica y el crecimiento en un país. (Jones, 1985) 


\section{Clasificación del Ahorro}

El ahorro se puede clasificar en ahorro privado y en ahorro público.

El ahorro privado es aquel que realizan las organizaciones privadas que no pertenecen al estado (básicamente familias, instituciones sin fines de lucro y empresas).

El ahorro de una empresa privada autónoma, equivale a su beneficio, menos la parte de éste que es repartida a sus propietarios o accionistas en forma de dividendos o participación en beneficios. El ahorro de las familias es igual a la renta disponible familiar menos el consumo privado y los impuestos.

El ahorro público lo realiza el estado, el cual también recibe ingresos a través de impuestos y otras actividades, a la vez que gasta en inversión social, en infraestructura (carreteras, puentes, escuelas, hospitales, etc.), en justicia, en seguridad nacional, etc. Cuando el Estado ahorra quiere decir que sus ingresos son mayores que sus gastos y se presenta un superávit fiscal, el caso contrario conduciría a un déficit fiscal.

También se puede ahorrar invirtiendo en diversos activos como pueden ser, bienes inmuebles, instrumentos de inversión. (Tipos de Ahorro, 2014)

\section{Ahorro y Política Económica}

El ahorro se encuentra influido por las políticas económicas que el Estado de un país tenga (éste puede afectar las tasas de interés y otras variables que afectan el ahorro). Los cambios en la tasa de interés, por ejemplo, pueden hacer que los ahorradores se sientan motivados o desmotivados a ahorrar. Un aumento en las tasas de interés puede hacer que los ahorradores tengan más razones para disminuir su consumo y ahorrar, o bien puede tener el efecto opuesto.

A iguales niveles de ingreso, depende de dos efectos conocidos como efecto ingreso y efecto sustitución. Así como el aumento de las tasas de interés puede incentivar a los ahorristas a consumir menos para ahorrar más, puede suceder que, al ser mayor el rendimiento del ahorro, se pueda cumplir la meta de acumulación prevista destinando una porción mayor del ingreso al consumo presente. Esta alza en las tasas de interés se puede deber, por ejemplo, a las formas en que el gobierno obtiene los recursos para sus actividades. Si el gobierno decide pedir recursos prestados al sistema financiero en una cantidad importante, las tasas de interés subirán. (SHCP, 2014) 


\section{El Ahorro en Latinoamérica y las Potencias Mundiales}

Debido a los bajos índices de ahorro interno que presentan los países latinoamericanos $(19,2 \%$ del PIB en promedio en 1994, contra 34,0\% en Asia), se ha dependido históricamente del ahorro externo para financiar sus necesidades de inversión. Los capitales externos se han manifestado en nuestras economías a través de diferentes vehículos en diferentes épocas.

Primero vinieron inversiones directas de parte de grandes multinacionales del sector primario (mineras, petroleras, agrícolas, etc.) y emisiones de bonos estatales en los mercados europeos. Luego, durante la posguerra y culminando en la crisis de la deuda de los ochentas, aunados a inversiones directas de empresas industriales, llegaron los créditos de la banca multilateral y comercial. Finalmente, a partir de 1990, se logró recuperar el acceso a los mercados de capitales, emitiendo títulos de renta fija y acciones de entidades estatales y privadas. Las reformas económicas de los últimos años, acompañadas de una mayor estabilidad económica y política en la región, han llevado a que el universo de inversionistas y prestamistas externos con exposición a Latinoamérica sea hoy más amplio que nunca. Estos inversionistas se pueden clasificar, principalmente, en dos categorías, dependiendo de su horizonte de inversión. (SALAS, 2003)

El ahorro es uno de los motores que impulsarán el crecimiento en los próximos años. Las tasas de ahorro privado descenderán en la mayoría de los países durante los próximos 50 años debido al envejecimiento de la población. "Sin embargo, la tasa de ahorro global se mantendrá estable hasta 2030 por la acción combinada de alto ahorro en China y la India". También el aumento del ahorro público de los países desarrollados para neutralizar el fuerte aumento de la deuda servirá para impulsar el crecimiento en los próximos años. (González, 2012)

\section{Política Fiscal y Ahorro}

Toda inversión ha de financiarse con ahorro, que, en su mayoría, procede de la economía nacional, mientras que el ahorro externo se limita, normalmente, a complementar el ahorro interno.

Dentro de la economía nacional, el sector privado es la fuente principal de ahorro. Las transferencias a los hogares, incluidos los pagos de prestaciones sociales, son el cauce principal a través del cual el gasto público afecta a la acumulación de ahorro privado. 
En este contexto, hay que tener presente que las transferencias podrían tener un efecto negativo sobre el ahorro. (Vásquez, 2011)

Por ahora, las pensiones constituyen la partida más importante de los sistemas públicos de prestaciones y, de mantenerse las políticas actuales, se prevé un considerable aumento del gasto para el futuro. El retraso de la jubilación efectiva y la introducción de nuevos planes de pensiones basados en mayor grado en el régimen de capitalización conllevarían un mayor nivel de ahorro en relación con el segmento de población de mayor edad, que podría tener un impacto positivo sobre el ahorro agregado.

Con todo, parece razonable llegar a la conclusión de que, a juzgar por la evidencia empírica, los elevados impuestos que financian sectores públicos de gran magnitud suelen afectar negativamente al ahorro y, en consecuencia, a la inversión y al crecimiento. Al igual que los impuestos sobre las rentas del trabajo desincentivan el empleo, los impuestos sobre el ahorro tienden a desincentivar éste y a estimular el consumo. Especialmente los impuestos sobre las sociedades, aunque también otros impuestos sobre las rentas del capital, son probablemente la causa de una disminución del ahorro privado en la medida en que reducen su rendimiento neto. (Soto, 2006)

\section{Relación Pib -Ahorro}

El ahorro nacional es la suma del ahorro público y el privado. El ahorro nacional viene dado por la diferencia entre la renta nacional o valor del conjunto de bienes producidos y el consumo:

$$
A=\mathrm{PIB}-C \quad \Leftrightarrow \quad \text { ahorro }=\mathrm{PIB}-\text { consumo }
$$

De hecho, este ahorro puede relacionarse con el consumo nacional (C), la cantidad de bienes exportados (X), la cantidad de bienes importados (M) y la cantidad de inversión (I) en la identidad fundamental de la contabilidad nacional:

$$
A-I=X-M \quad \Leftrightarrow \quad \text { ahorro }- \text { inversiones }=\text { exportaciones }- \text { importaciones }
$$


Esta igualdad se deduce de la propia definición del Producto Interno Bruto (PIB) evaluado a precios de mercado (BancaFácil, 2014).

$$
Y=\mathrm{PIB}_{p m}+M=C+I+X
$$

\section{Planteamiento del Problema}

\section{La gestión del ahorro en el Ecuador}

La elección entre consumo y ahorro es la elección entre consumo presente y consumo futuro, y es una de las decisiones en materia económica que más impacto tiene sobre el nivel de vida de los habitantes que la realizan.

Una exagerada preferencia por el consumo actual, patente en las expansiones populistas en países como el Ecuador ha registrado en su historia reciente, tiene su contrapartida en una acumulación neta de pasivos externos (acumulación de deuda externa y/o disminución en las reservas internacionales netas), en una disminución en el ritmo de acumulación de capital (disminución en la inversión doméstica) o en una combinación de ambas. Esta preferencia por el consumo actual conlleva, a través de estos mecanismos, a una disminución en la capacidad futura de generar ingresos y lleva implícita la imposición de un límite sobre el nivel de vida de las futuras generaciones. Por otro lado, una exagerada preferencia por el consumo futuro (ahorro actual) puede implicar una reasignación de recursos hacia proyectos de inversión que rindan menos utilidad que aquella que se sacrifica en el presente. En este caso, la decisión también resultaría sub-óptima y, contrariamente al caso anterior, las generaciones actuales son las que estarían financiando un nivel de bienestar superior para las generaciones futuras.

En los últimos años, la economía ecuatoriana ha experimentado cambios sustanciales que habrían redundado en una cierta sensación de progreso entre los agentes económicos. La estabilidad económica y social ha creado el clima propicio para el desarrollo de la inversión privada, que en los últimos tres años se ha expandido a una tasa real que triplica la ya alta tasa de crecimiento del producto bruto interno (PBI). Al mismo tiempo, una mejora en las expectativas reflejada en la revalorización de los activos nacionales, ha contribuido a que las percepciones de riqueza o de ingreso permanente de los agentes aumenten. (GestioPolis, 2015) 
Como han influido las reformas económicas y políticas de los últimos años, en la estabilidad económica y en las fuentes de ahorro, dentro del sector público y privado de la economía nacional y global como se ha gestionado

Conocer cómo enfrentar obligaciones futuras y tener recursos disponibles con los cuales resistir variaciones en la economía.

Buscar mecanismos con un nivel de ahorro adecuado para potenciar beneficio en crisis económicas.

Tener un adecuado balance entre ahorro y rendimiento, un plan que se realice en un periodo acotado de tiempo.

Predisponer de un ahorro cotidiano líquido y disponible de acuerdo a los cambios de la economía.

\section{Metodología}

La poca accesibilidad que presenta el área de estudio, ya que es una investigación global, el ajustado tiempo que se tiene para realizar la investigación, las limitaciones en materia de fuentes documentales y estadísticas que se tiene, por razones ya mencionadas, han dificultado el análisis de este tema. Cabe destacar, sin embargo, que algunos obstáculos citados se han podido salvar, a través, por ejemplo, de la consulta bibliográfica y estadística en Internet o la utilización de información de informes.

La metodología utilizada, como ya se ha comentado, debido al carácter aplicado de la investigación, tiene bastante paralelismo con el esquema seguido en los estudios de análisis bibliográfico.

Se analizaron los resultados de la investigación obtenida a través de páginas web acerca del ahorro, por lo que se utilizó el método bibliográfico que, en un sentido amplio, es el sistema que se sigue para obtener información contenida en documentos. En sentido más específico, el método de investigación bibliográfica es el conjunto de técnicas y estrategias que se emplean 
para localizar, identificar y acceder a aquellos documentos que contienen la información pertinente para la investigación.

\section{Desarrollo}

\section{El Ahorro en la Economía Del Ecuador}

\section{Década de 1960 y Década de 1970}

El PIB per cápita de Ecuador a principios de los Años 60 fue de 222 dólares. A finales de la década (1979), Ecuador llegó a los 1.824 dólares, habiendo elevado en un 287,2\% su PIB per cápita con respecto a 1970, por lo que el ahorro con relación al PIB del año 1960 y 1970 fue mucho menor considerando el incremento que tuvo a finales de 1979, donde el consumo nacional por explotación de tierras productivas se vio involucrado. (FMI, 2013)

\section{Década de 1980 y Década de 1990}

El PIB per cápita de Ecuador a principios de los Años 80 fue de 2.097 dólares, El PIB per cápita de Ecuador a principios de los Años 90 fue de 1.173 dólares. A finales de la década (1999), Ecuador llegó a los 1.599 dólares, habiendo elevado en un 36,3\% su PIB per cápita con respecto a 1990. Luego de la crisis de 1999, durante el gobierno Jamil Mahuad al país le tomó 7 años volver a los niveles de bienestar de 1995, para después salir adelante. La crisis financiera durante el gobierno liberal de Jamil Mahuad produjo aproximadamente un $70 \%$ del cierre de las instituciones financieras del país. En 1999 la actividad económica fue -7 o -8\% y el sucre perdía su valor por 195\%. Las pérdidas económicas ascendieron a 8.000 millones de dólares. El ingreso per cápita del dólar americano había caído por 32\% durante aquel año. El desempleo aumentó de $9 \%$ al $17 \%$ y el subempleo aumentó de $49 \%$ al 55\%. Fueron utilizados 1.6 mil millones dólares de los fondos del Estado de Ecuador a los bancos que quebraron. El medio circulante aumentó a una proporción anual de $170 \%$ para pagar a los depositantes de los bancos quebrados. (BCE, 2013)

\section{Década de 2000 y Década de 2010}

El PIB per cápita de Ecuador a principios de los Años 2000 fue de 1.462 dólares. El PIB per cápita de Ecuador a principios de los Años 10 fue de 4.633 dólares. Hasta mediados de la década 
(2015), Ecuador llegó a los 6.250 dólares, habiendo elevado en un 34,0\% su PIB per cápita con respecto al año 2010. En enero de 2000 el gobierno decretó la dolarización a una paridad de 25.000 sucres por dólar. Por lo cual, quienes tenían ahorros congelados en sucres, recuperaron sólo una quinta parte.

A pesar del crecimiento vigoroso de los últimos años, y al igual que la mayoría de los países latinoamericanos, su economía sigue dependiendo de las exportaciones de materias primas y el petróleo es la principal fuente de riqueza del país. (INEC, 2011)

\section{Coyunturas a Nivel Mundial}

\section{El Ahorro en Europa}

Es difícil en pocas líneas explicar de forma fundamentada los efectos económicos y sociales que el déficit público, los recortes en el gasto de la administración o el gran tamaño que ha adquirido la deuda pública tienen en ciudadanos y empresas, una parte importante de los cuales se encuentra en una difícil situación. No obstante, merece la pena tratar de aportar puntos de vista que propongan mejoras a este escenario.

Uno de los gastos más importantes se refiere al pago de pensiones, seguros de desempleo y otras ayudas de tipo social, partida que supone cerca de un $40 \%$ del gasto público total. Es esencial abordar la reestructuración del sistema de pensiones y compensaciones por desempleo, no sólo con vista a reducir este importante gasto, sino a prever los efectos del envejecimiento de la población europea, que harán insostenible el sistema. Lo importante es realizar esta reestructuración atendiendo a criterios de solidaridad y redistribución de rentas que no afecten a las familias e individuos que mayor necesidad tienen de estas ayudas.

No es conveniente recortar más el gasto educativo y sanitario; una partida de gasto de la que se debate mucho, y que sin embargo representa sólo un 6\%, aproximadamente, del total. Lo que sí es perentorio, especialmente en materia de educación, es mejorar sensiblemente los resultados de estas políticas: una sociedad más sana y mejor educada en todos los aspectos (incluidas las disciplinas científicas) es más capaz de contribuir al crecimiento económico en el medio y largo plazo. 
Por último, la administración no debe actuar donde hay un sector privado preparado y eficiente que pueda desarrollar ciertas actividades a un menor coste, aunque siempre con el debido control si se trata de la prestación de servicios públicos. (Escobar, 2008)

\section{El Ahorro en Asia}

Para analizar el ahorro con más detalle, examinamos la evolución de las tasas de ahorro nacional bruto y la composición del ahorro en China, Corea e India. Estas tres economías representan alrededor de las tres cuartas partes del PIB de Asia (excluido Japón). Y las tres han experimentado un aumento del ahorro corporativo. En China, la proporción del ahorro corporativo registró un marcado aumento, y representó casi la mitad del ahorro nacional en 200708. Curiosamente, en India, el ahorro de los hogares sigue siendo la principal fuente de ahorro nacional, y representa alrededor del 20\% del PIB desde principios de 2000, mientras que el ahorro corporativo se ha convertido en una fuente cada vez más importante en los últimos años. En Corea, el ahorro de los hogares se ha reducido desde finales de los años noventa, lo que ha contribuido a un ligero descenso del ahorro nacional global.

Al examinar el ahorro con respecto al ingreso disponible se observa una perspectiva diferente del ahorro nacional. La tasa de ahorro de los hogares en India registró un fuerte aumento en los últimos diez años, del 20\% del ingreso disponible en 1998 al 32\% en 2008. De hecho, India parece registrar ahora la tasa más alta de ahorro nacional de las economías de Asia sobre las que se dispone de datos. En cambio, la tasa de ahorro de los hogares de Corea se ha reducido considerablemente, de casi 30\% a finales de los noventa a 7\% en 2007. Tanto la evolución de las tasas de ahorro global como las fuentes del ahorro nacional varían sustancialmente de un país a otro. China representó alrededor del 62\% del ahorro nacional bruto de toda Asia (excluido Japón) en 2008. En términos de magnitud, el fuerte aumento del ahorro corporativo y la evolución del ahorro en China influyen claramente en la evolución del ahorro global en Asia. (Prasad, 2009)

\section{El Ahorro en Estados Unidos}

Durante el último decenio, el ahorro de las familias en Estados Unidos ha disminuido más de lo que el ahorro público (expresado por los excedentes presupuestarios) ha aumentado en el mismo periodo. El enorme déficit del balance de pagos de las transacciones corrientes de Estados Unidos, de alrededor del 4,5\% del PNB en el año 2000, refleja ese desequilibrio del ahorro. Para 
financiar un nivel normal de inversión interior -históricamente alrededor del 17\% del PNB- los Estados Unidos han debido utilizar ampliamente el ahorro del resto del mundo. "Malas" reducciones de impuestos - las que reducen el ahorro público sin estimular el ahorro privado podrían incrementar esa deuda con el extranjero. "Desde hace más de veinte años, los Estados Unidos recurren ampliamente a las reservas limitadas del ahorro mundial para sostener su alto nivel de consumo - la de la administración federal en los años 1980 y la de las familias en los años 1990. Las entradas netas de capitales son actualmente más importantes que en el conjunto de los países en desarrollo. Es así como Estados Unidos, que era acreedor del resto del mundo a comienzos de 1980, se ha convertido en el más grande deudor mundial: unos 2 billones 300 mil millones de dólares en el año 2000. "Los balances de las familias y de las empresas en Estados Unidos muestran el efecto acumulado de los préstamos privados obtenidos en el exterior desde hace diez años. La deuda de las familias alcanza ahora un record de 1,1 veces los ingresos disponibles y la deuda de las empresas es también muy elevado con relación a su flujo de fondos. (Teitelbaum, 2013)

\section{El ahorro en Ecuador y su relación con el contexto internacional}

Tradicionalmente la economía ecuatoriana se ha sustentado en la agricultura, la minería y la pesca. Desde los años setenta la industria petrolera ocupó un papel vital en el desarrollo del país, pero a partir de este siglo, las exportaciones de productos agrícolas son el verdadero motor de crecimiento económico del país. Según los datos macroeconómicos, Ecuador está en un constante crecimiento, su producto interior bruto se ha visto triplicado en los últimos 10 años, en la misma línea ascendente se encuentra su renta per cápita que se ha incrementado un $235 \%$ en el mismo período, a la par con el ahorro que va incrementando, las reformas económicas de los últimos años, acompañadas de una mayor estabilidad económica y política en la región, han llevado a que el universo de inversionistas y prestamistas externos con exposición a Latinoamérica sea hoy más amplio que nunca, dentro de la economía nacional, el sector privado es, con mucho, la fuente principal de ahorro. (MUNDIAL, 2014).

\section{Resultados}

Un programa de ahorro se presenta como los pasos sistemáticos para la obtención de resultados, así como estrategias que deben seguirse y acciones principales que deben ejecutarse a fin de 
lograr o rebasar los objetivos establecidos; en este sentido se presenta a través de la conservación y administración del dinero, que es el uso racional y efectivo del mismo para maximizar beneficios y destacar las situaciones competitivas. De hecho, cualquier actividad que conlleve al uso racional y juicioso del dinero, nivelación de demanda para minimizar las facturas se consideran administración de dinero (ahorro).

\section{El objetivo principal del ahorro es el mejoramiento continuo del beneficio y la intensificación en la reducción de gastos.}

De acuerdo al problema planteado, planteamos un programa de ahorro económico, se incorporó el tipo de investigación denominado Proyecto Factible. El mismo consiste, en una proposición sustentada en un modelo operativo factible, orientada a resolver un problema planteado o a satisfacer necesidades en un campo de interés. Esta modalidad se presenta por la necesidad de incorporar una solución al problema del alto consumo de bienes o servicios, y así garantizar que la misma ofrezca una minimización de costos y gastos. Dicha minimización incluye estrategias oportunas, eficientes y eficaces para asegurar la continuidad de una manera satisfactoria y beneficiosa.

Al analizar los datos arrojados por esta investigación en un plan global de investigación que integra de un modo coherente y adecuadamente correctas técnicas de recogida de datos a utilizar, análisis previstos y objetivos, el diseño de una investigación intenta dar de una manera clara y no ambigua respuestas a las preguntas planteada en la misma. Tomándose en cuenta los objetivos propuestos para este proyecto se consideró una investigación de campo, ya que permite no sólo observar, sino recolectar los datos directamente de la realidad objeto de estudio.

\section{Como propuesta de este estudio documental se plantea un Programa De Ahorro Económico}

Tal como se realizó un diagnóstico en análisis de datos, se busca presentar un programa de ahorro, no a nivel global, porque es casi imposible implementarlo en todo el mundo, pero si ser una opción más considerable para ahorrar.

Establecer un presupuesto (con un objetivo a alcanzar)

Es importante que tengamos en cuenta los gastos fijos mensuales para calcular una meta de ahorro. 
Comparar precios y buscar ofertas:

Probablemente cuando conozcamos lo que se ahorran los demás, nos empecemos a interesar más en lo que gastamos cada mes.

Evitar realizar gastos por impulso (casi nunca lo necesitas)

El consumismo impulsivo es el peor enemigo del ahorro y puede alterar el presupuesto sin que nos demos cuenta.

\section{Conclusiones}

El consumo actual de dinero para cubrir las necesidades es más de lo que debería por tal razón no se permite capitalizar un porcentaje muy alto para ahorrar.

Una vez finalizado el proyecto, se puede denotar que es posible ahorrar, en esta investigación se describen unas opciones de mejora en la eficiencia económica alternativas, y se propone un método para llevar a cabo estas aplicaciones.

Después de efectuar las diferentes comparaciones entre las potencias mundiales se puede decir que el manejo político les ayuda mucho en su economía y mantener una reserva de dinero para afrontar emergencias o necesidades.

La política administrativa en Ecuador ha influenciado mucho para que su economía no esté en buen auge y las reservas estatutarias de dinero no sean las adecuadas.

\section{Se recomienda}

La concientización y racionalización en el uso de dinero.

La reducción de gastos, sustituyendo gastos en inversiones.

Implementar una campaña de concientización para el uso racional del capital tanto personal como nacional.

En las áreas donde el análisis económico arroje una recuperación acorde con las expectativas de las inversiones, realizar un plan más eficiente de ahorro. 


\section{Referencias Bibliográficas}

(2014). Tipos de Ahorro. México.

BancaFácil. (2014). Ahorro-PIB.

BCE. (2013). PREVISIONES MACROECONÓMICAS 2000. Quito: BCE.

BLANCO, I. (1998). Nomenclatura y principales conceptos de economía. LIMA: TORRE DE

\section{BABEL EDICIONES.}

Escobar, M. J. (2008). Ahorro Público EY-España. Barcelona: La Razón.

FMI. (2013). PIB PER CÁPITA.

GestioPolis. (2015). Ahorro Nacional. Quito.

González, J. S. (09 de 11 de 2012). EL PAÍS (ECONOMÍA). Obtenido de http://economia.elpais.com/economia/2012/11/09/actualidad/1352456628_222082.html

INEC. (2011). CUENCA: CEPAL.

Jones, H. (1985). En Introducción a las teorías modernas del crecimiento económico. Barcelona.

MUNDIAL, F. E. (2014). COMPETITIVIDAD DE ECUADOR CON EL MUNDO.

Prasad, E. (2009). Reequilibrio del Crecimiento en Asia. Washington, D.C...

SALAS, X. (2003). APUNTES DE CRECIMIENTO ECONÓMICO. CHILE.

SHCP, C. Y. (2014).

Soto, M. J. (2006). Economía ahorro e inversión. LIMA.

Spencer, M. H. (1998). Introducción a la teoría económica. En M. H. Spencer, Economía Contemporánea. Barcelona: REVERTE S.A.

Superintendencia, d. B. (s.f.). Banca Fácil. Obtenido de http://www.bancafacil.cl/bancafacil/servlet/Contenido?indice=1.2\&idPublicacion=15000000000 00022\&idCategoria $=4$

Teitelbaum, A. (2013). Sistema Financiero Mundial. Chicago.

Vásquez, A. (2011). Ahorro Económico. 